\title{
ASPET Journals Support the National Institutes of Health Principles and Guidelines for Reporting Preclinical Research
}

Over the last several years there has been growing concern within the biomedical research community regarding a common perception that the level of scientific reproducibility and self-correction in preclinical research is not adequate (Collins and Tabak, 2014). In one initiative to address this situation, the National Institutes of Health (NIH) in collaboration with Nature and Science/American Association for the Advancement of Science held a workshop on June 14, 2014, for biomedical journal editors (including The Journal of Pharmacology and Experimental Therapeutics) that addressed the reproducibility and rigor of published research findings. Workshop attendees provided input to a draft set of methodological guidelines for published research. The ASPET Board of Publications Trustees reviewed the draft guidelines and submitted a response to the NIH on August 19, 2014.

On November 5, 2014, the National Institutes of Health announced "Principles and Guidelines for Reporting Preclinical Research" (http://www.nih.gov/about/reporting-preclinical-research.htm). The guidelines address five principle areas of concern for the publication of preclinical biological research: rigorous statistical analysis, transparency in reporting, data and material sharing, consideration of refutations, and the establishment of best practice guidelines in the areas of image-based data and the description of biological materials with enough information to uniquely identify the reagents (in particular, antibodies, cell lines, and animals).

ASPET publishes three primary research journals: The Journal of Pharmacology and Experimental Therapeutics (JPET; founded in 1909), Molecular Pharmacology (1965), and Drug Metabolism and Disposition (DMD; 1973). ASPET also publishes a review journal, Pharmacological Reviews, established in 1949. It is the intent and mission of ASPET's journals to hold to high standards of scientific rigor. Since their inception, ASPET's primary research journals have placed no limit on the Materials and Methods section in each journal, allowing for detailed descriptions of experimental methodology. Critical evaluation of experimental design issues, statistical analyses, and the validation of reagents have also been key components of the peer-review process. Peer reviewers are evaluated by the editors-in-chief and associate editors on their attention to these issues. In recent years, ASPET's Board of Publications Trustees has expanded and strengthened its reporting requirements. For instance, the chemical identity of novel research compounds must be provided, and the sex of experimental subjects must be noted for in vivo studies and studies using primary cultures of cells or tissues from animals or humans. The coordinates and structure factor amplitudes for studies, including crystallographic data, must be provided and deposited with the Protein Data Bank. The nomenclature used to identify receptors and ion channels should conform to the guidelines of the Committee on Receptor Nomenclature and Drug Classification of the International Union of Basic and Clinical Pharmacology (NC-IUPHAR), whose nomenclature reports are published in Pharmacological Reviews. Most recently, we eliminated limits on references.

Consistent with its history, ASPET strongly supports the NIH Principles and Guidelines for Reporting Preclinical Research. ASPET's journals and over 70 other leading biomedical research journals have endorsed these guidelines (http://www.nih.gov/about/endorsingjounals.htm). In order to further enhance support for the goals of the NIH guidelines and provide a higher level of transparency in the areas of experimental design, description of methods, and statistical analysis, ASPET has implemented the following editorial actions: The DMD, JPET, and Molecular Pharmacology Web sites have been updated 
with revised Instructions to Authors establishing the preferred standards for reporting of experimental design and methodology and reporting requirements for randomization, blinding, normalization and statistical analyses, and reagent validation. Several excellent reviews, commentaries, and the references therein on key aspects of experimental design and statistical analysis have been recently published that authors and readers will find of value (Landis et al., 2012; Marino, 2014; Motulsky, 2014). The DMD, JPET, and Molecular Pharmacology Editorial Boards will carefully review how authors have addressed these methodological parameters during the peer-review process for all future submissions to the journal in an effort to further enhance the scientific rigor and transparency of pharmacological research reported in DMD, JPET, and Molecular Pharmacology. These changes were developed by the Board of Publications Trustees working with the editors of the ASPET journals. We know that you, our readers and authors, are proud to join us in this goal of increased research quality and transparency.

ASPET Board of Publications Trustees
Mary Vore, Chair
Darrell Abernethy
Randy Hall
Michael Jarvis
Kathryn Meier
Edward Morgan
Kim Neve
David Sibley
Stephen Traynelis
Jeffrey Witkin
Judy Siuciak, ex officio

\section{References}

Collins FS and Tabak LA (2014) Policy: NIH plans to enhance reproducibility. Nature 505:612-613.

Landis SC, Amara SG, Asadullah K, Austin CP, Blumenstein R, Bradley EW, Crystal RG, Darnell RB, Ferrante RJ, Fillit H, et al. (2012) A call for transparent reporting to optimize the predictive value of preclinical research. Nature 490:187-191.

Marino MJ (2014) The use and misuse of statistical methodologies in pharmacology research. Biochem Pharmacol 87:78-92.

Motulsky HJ (2014) Common misconceptions about data analysis and statistics. J Pharmacol Exp Ther 351:200-205.

dx.doi.org/10.1124/pr.115.011205. 